\title{
Serologische Influenza-Diagnostik: Ist der Immunofluoreszenztest eine Alternative zum Hämagglutinationshemmtest beim stammspezifischen Antikörpernachweis?
}

\author{
G. Döller, C. Merk, H.-J. Gerth
}

Hygiene-Institut, Tübingen

\section{Zusammenfassung:}

Auslösend für diese Studie war die im August bekannt gewordene Beobachtung, daß in Asien Influenza A-Viren des Subtyps H1N1, A/Singapore/6/86, isoliert worden waren, die im Hämagglutinationshemmtest (HAH) deutliche Unterschiede gegenüber den bis dahin bekannten H1N1-Stämmen (letzte Impfempfehlung A/Chile/1/83), aufwiesen.

Es sollte untersucht werden, inwieweit die Ergebnisse unseres Immunfluoreszenztests, bei dem mit Virusantigen und mit Kontrollantigen beladene Erythrozyten (IFTE) verwendet werden, hinsichtlich einer stammspezifischen Reaktivität mit den Ergebnissen des $\mathrm{HAH}$ vergleichbar sind.

Ein Vergleich von IFTE und HAH mit spezifischen Antiseren (vom Huhn) erbrachte identische stammspezifische Ergebnisse, jedoch müssen bei dem HAH unspezifische Seruminhibitoren inaktiviert werden.

Die Untersuchung von 13 Seren von 11 Patienten, die Anfang 1987 an einer Influenza A/H1N1-Infektion erkrankt waren, machte deutlich, daß eine stammspezifische Diagnostik nur begrenzt möglich ist. Da die Influenza-Aktivität in unserem Einzugsgebiet Anfang 1987 gering war, mußte die Versuchsreihe auf die o.g. Seren beschränkt werden. In einer zweiten Versuchsreihe wurden 30 Seren von 16 Patienten aus einer Influenza A/H3N2-Epidemie, die vier Jahre vor der Isolierung des heterologen Stammes A/Leningrad/360/ 86 lag, verwendet. Mit dem IFTE wurde bei allen Patientenseren eine bevorzugte Antikörperantwort für den damals kursierenden Stamm (A/Bangkok/1/79 ähnlich) sowohl im IgG-als auch im lgA-Bereich festgestellt. Hingegen konnte bei 4 von 16 Patienten im HAH überhaupt kein Stammesunterschied festgestellt werden, bei 11 Patienten betrug der Titerunterschied nur eine Titerstufe.

Schlüsse/wörter:

Influenza $A$ und $B$ - Subtyp- und Immunglobulinklassen-spezifischer IFT - HAH - Stammspezifitätsprüfung

\section{Summary:}

This study was initiated by the appearance of influenza A/H1N1-strains in 1986 (prototype strain A/Singapore/6/86) which showed considerable antigenic differences to their predecessors (prototype strain $A /$ Chile/1/83) in the hemagglutination inhibition test (HIT). The aim of this study was to compare the results of an immunofluorescence test based on antigen-loaded erythrocytes (IFTE) with that of the HIT in regard to strain specificity.

Comparison of the IFTE with the HIT using specific antisera obtained from chicken after immunisation with allantoic fluid antigens yielded equivalent results.

Though only a small number of sera from patients recently infected have been available in 1987, the examination of 13 sera from 11 patients showed the limitations of strain specific influenza diagnosis in immunologically primed patients. Very similar results were obtained regardless of which method was used. In a second experiment results of IFTE and HIT were compared in a series of 30 sera obtained from 16 patients infected with influenza A/H3N2 in 1983. A/Bangkok/1/79 (recommended for vaccination in 1982/ 83) and $A /$ Leningrad/360/86 (recommended for vaccination in 1987/88) served as antigens. By IFTE all sera showed a significantly greater antibody response to the earlier strain. In contrast, in HIT 4/16 patients reacted as well with the later strain or showed only a 2fold higher titer with the earlier than with the later strain.

Keywords:

Influenza A and B - IFTE - HIT - strain-specificity 


\section{Einleitung}

Die Subtyp- und Immunglobulinklassen-spezifische serologische Influenza-Diagnostik ist durch die Entwicklung eines Immunfluoreszenztests auch für die Routinediagnostik ermöglicht worden (1 - 3) Im Gegensatz zum IFTE erlaubt die bisher im Routinelabor an häufigsten verwendete Komplementbindungsreaktion (KBR) nur. eine Typ-spezifische serologische Diagnostik. Mit dem konventionellen 'Hämagglutinationshemmtest. (HAH) hingegen kann eine Stamm-differenzierende InfluenzaDiagnostik durchgeführt werden. Der $\mathrm{HAH}$ ist jedoch zeitaufwendig und störanfällig, ganz besonders dựch die Anwesenheit unspezifischer Seruminhibitoren, die eine Interpretation der Testergebnisse erschwereen ôder unmöglich maçhen.

\section{Problemstellung}

Auslösend für diese Studie war die im August 1986 bekannt gewordene Beobachtung, daß in Asien Influenza A-Viren des Subtyps H1N1, die deutliche Unterschiede gegenüber den bis dahin bekannten $\mathrm{H} 1 \mathrm{~N} 1$ Stämmen aufwiesen, isoliert worden waren. Insbesondere der damals im Impfstoff enthaltene Stamm A/Chile/ 1/83 ( $\mathrm{H} 1 \mathrm{~N} 1$ ) zeigte im Hämagglutinationshemmtest (HAH) kaum mehr serologische Verwandtschaft mit diesen neuen Stämmen. Es sollte daraufhin ermittelt werden, in welchem Maße der Immunfluoreszenztest mit virusbeschichteten Erythrozyten (IFTE) stammspezifisch reagiert. Dazu wurden die Influenza-Impfstämme, die in dem Impfstoff für die Saison 1986/87 und 1987/88 empfohlen worden waren, verwendet und spezifische Antikörper im Huhn hergestellt. Für Influenza B wurde der Stamm aus der Impfempfehlung 1985/86 in die Studie einbezogen. Zur Auswertung des stammdifferenzierenden Potentials des IFTE bei natürlichen Influenza A/H3N2-Infektionen des Menschen wurde der Stamm A/Bangkok/1/79, der in der Impfempfehlung für 1982/83 enthalten war, zu dieser Studie verwendet. Es wurde die Möglichkeit des Nachweises einer stammspezifischen Immunantwort mittels des IFTEs an Seren von Patienten mit InfluenzaInfektionen im Zeitraum 1982-1987 untersucht.$$
\text { - - }
$$

\section{Materialien und Methoden}

\section{Influenzastämme und deren Vermehrung}

Folgende Influenzastämme wurden in 10 Tage bei $37^{\circ} \mathrm{C}$ bebrüteten embryonierten Hühnereiern vermehrt:

Influenza A/Bangkok/1/79 (H3N2), Abkürzung: A/ Bang.

Influenza A/Mississippi/1/85 (H3N2), Abkürzung: A/ Miss.

Influenza A/Leningrad/360/86 (H3N2), Abkürzung: A/ Leningr.

Influenza $A / C h i l e / 1 / 83$ (H1N1), Abkürzung: $A / C h i l e$.

Influenza A/Singapore/6/86 (H1N1), Abkürzung: A/ Sing.

Influenza $B /$ USSR/100/83, Abkürzung: B/USSR.

Influenza $B / A n n$ Arbor/1/86, Abkürzung: $B / A A$.

Alle Influenzastämme wurden vom WHO International Laboratory for Biological Standards, Hertfordshire, EN6 3QG, England, erhalten. Nach Ernte der virushaltigen Allantoisflüssigkeit wurde diese niedertourig (2000 Upm,
$30 \mathrm{~min}, 4^{\circ} \mathrm{C}$ ) zentrifugiert und im Hämagglutinationstest (HA) nach einer Standardmikromethode (4) mit einer $0,5 \%$ igen Hühnererythrozytensuspension titriert. Die reziproken HA-Titer betrugen für:

$\begin{array}{ll}\text { A/Bang. } & : 256 \\ \text { A/Miss. } & : 512 \\ \text { A/Leningr. } & : 512 \\ \text { A/Chile } & : 1024 \\ \text { A/Sing. } & : 1024 \\ \text { B/USSR } & : 128 \\ \text { B/AA } & : 128 .\end{array}$

\section{Gewinnung der Antiseren vom Huhn}

Wèißen Leghorn-Hybriden wurde die virushaltige Allantoisflüssigkeit óhne Zusatz von Adjuvvantien intramuskulär an vier verschiedenen Ștellen verabreicht, für Influenza A/H3N2-Stämme $1 \mathrm{ml}$ Volumen und für Influenza A/ H1 N1 - und für Influenza B-Stämme ca. $4 \mathrm{ml}$. Alle Hühner erhielten nach 4 Wochen eine weitere intramuskuläre Injektion von 1 bis $1,5 \mathrm{ml}$ Virussuspension. Die Serumabnahme erfolgte vor Immunisierung und dann 45 bis 53 Tage nach Immunisierungsbeginn.

\section{Immunfluoreszenz̈test}

\section{Untersuchung der Antiseren vom Huhn}

Für die Untersuchung der gewonnenen Antiseren wurde ein für das Hühnersystem modifizierter Immunfluoreszenztest (IFTE) verwendet, wobei mit Antigen- und mit Kontrollantigen beladene Erythrozyten auf Objektträger gebracht wurden (3). Nach Fixierung mit Aceton wurden $10 \mu \mathrm{l}$ entsprechend verdünntes Hühnerserum/Objektträgerfeld $2 \mathrm{Std}$. bei $37^{\circ} \mathrm{C}$ in einer feuchten Kammer inkubiert. Die Verdünnungsflüssigkeit bestand aus $20 \%$ Kälberserum/PBS. Nach Abwaschen der Objektträger mit destilliertem Wasser wurden diese unter einem Fön getrocknet und $10 \mu \mathrm{l}$ anti-Huhn-lgG (FITC markiert, Fa. Wellcome Diagnostics, Dartford, England, $1: 40$ verdünnt in $20 \%$ Kälberserum/PBS und Evans blue, $1: 10000$ ) pro Objektträgerfeld aufgegeben und für $1 \mathrm{Std}$. bei $37^{\circ} \mathrm{C}$ in einer feuchten Kammer inkubiert. Nach Waschen der $\mathrm{Ob}$ jektträger mit destilliertem Wasser wurden die Objektträger unter einem Auflichtfluoreszenzmikroskop bei 250facher Vergrößerung (Wasserimmersion) ausgewertet.

\section{Untersuchung der Patientenseren}

Die Untersuchung der Patientenseren erfolgte mit einem IFTE, bei dem mit Antigen- und mit Kontrollantigen beladene Erythrozyten verwendet werden. Der Test wurde bereits ausführlich beschrieben (3).

\section{Hämagg/utinationshemmtest}

Der Hämagglutinàtionshemmtest (HAH) wurde nach einer Standardmikromethode durchgeführt (4). Es wurden die im Brutei hergestellten Antigene verwendet, wobei die Influenza B-Stämme mit Äther gespalten wurden. Zur Entfernung unspezifischer Seruminhibitoren wurden sämtliche Seren mit Na-Perjodat oder mit Test-Neuraminidase von V. cholerae (Fa. Behringwerke AG, Marburg) inaktiviert.

\section{Komplementbindungsreaktion}

Die Komplementbindungsreaktion (KBR) wurde mit kommerziell erhältlichem Influenza A-Antigen für die 
KBR (Fa. Behringwerke AG, Marburg) nach einer Standardmikromethode durchgeführt (7).

\section{Patientenseren}

Die Patientenseren stammten entweder aus dem diagnostischen Labor der Abteilung für Medizinische Virologie und Epidemiologie der Viruskrankheiten des HygieneInstituts Tübingen oder von Herrn Dr. Bernd Stekeler, Praxis für Allgemeinmedizin. Hechingen. Die Seren wurden in folgenden Zeiträumen gesammelt (Tab.3): Das Serum des Patienten 1 wurde am 22. 2. 1986 abgenommen, die übrigen Seren im Januar und Februar 1987. Tab. 4 und 5: Die Serumabnahmen erfolgten zwischen Januar 1983 und März 1983. Da für die Impfung 1982/ 83 Influenza A/Bangkok/1/79 (H3N2) empfohlen war, wurde dieser Stamm in die Studie einbezogen.

\section{Ergebnisse}

Auf Tab. 1 sind die Ergebnisse dargestellt, die wir mit dem Kreuz-Immunfluoreszenztest erhalten haben.

Tab. 2 zeigt die Ergebnisse der hämagglutinationshemmenden Antikörper im Kreuz-Hämagglutinationshemmtest. Aus den Tabellen ist ersichtlich, daß sowohl HAH als auch IFTE stammspezifisch reagieren.

Auf Tab. 3 sind vergleichend Komplementbindungsreaktion- (KBR), IFTE- und HAH-Titer von 13 Patientenseren dargestellt. Die Patienten waren an einer Influenza A/ H1N1-Infektion erkrankt gewesen. Patient 1 hatte sehr wahrscheinlich eine Influenza A/Sing.-Infektion, was deutlich sowohl durch den hohen IgG- und IgA-Titer im IFTE als auch in dem hohen HAH-Titer von 1024 zum Ausdruck kam, eine Kreuzreaktion mit A/Chile war so-

Tab. 1: IgG-Antikörper (Huhn) im Kreuz-Immunfluoreszenztest

1. = vor Immunisierung

2. = 45-53 Tage nach Immunisierungsbeginn

\begin{tabular}{|c|c|c|c|c|c|c|c|}
\hline \multirow{2}{*}{$\begin{array}{l}\text { Antiserum } \\
\text { (Huhn) }\end{array}$} & \multirow{2}{*}{$\begin{array}{l}\text { *Ab- } \\
\text { nahme }\end{array}$} & \multicolumn{6}{|c|}{ Referenzstämme } \\
\hline & & $\begin{array}{l}\text { A/Miss. } \\
\text { (H3N2). }\end{array}$ & $\begin{array}{l}\text { A/Leningr. } \\
\text { (H3N2) }\end{array}$ & $\begin{array}{l}\text { A/Chile } \\
\text { (H1N1) }\end{array}$ & $\begin{array}{l}\text { A/Sing. } \\
\text { (H1N1) }\end{array}$ & B/USSR & B/AA \\
\hline $\begin{array}{l}\text { A/Miss. } \\
\text { (H3N2) }\end{array}$ & $\begin{array}{l}1 . \\
2 .\end{array}$ & $\begin{array}{l}<40 \\
2560\end{array}$ & $\begin{array}{l}<40 \\
1280\end{array}$ & $\begin{array}{l}<40 \\
<40\end{array}$ & $\begin{array}{r}<40 \\
40\end{array}$ & $\begin{array}{l}<40 \\
<40\end{array}$ & $\begin{array}{l}<40 \\
<40\end{array}$ \\
\hline $\begin{array}{l}\text { A/Leningr. } \\
\text { (H3N2) }\end{array}$ & $\begin{array}{l}1 . \\
2 .\end{array}$ & $\begin{array}{r}<40 \\
1280\end{array}$ & $\begin{array}{l}<40 \\
2560\end{array}$ & $\begin{array}{l}<40 \\
<40\end{array}$ & $\begin{array}{l}<40 \\
<40\end{array}$ & $\begin{array}{l}<40 \\
<40\end{array}$ & $\begin{array}{l}<40 \\
<40\end{array}$ \\
\hline $\begin{array}{l}\text { A/Chile } \\
\text { (H1N1) }\end{array}$ & $\begin{array}{l}1 . \\
2 .\end{array}$ & $\begin{array}{r}<40 \\
40\end{array}$ & $\begin{array}{l}<40 \\
<40\end{array}$ & $\begin{array}{r}<40 \\
640\end{array}$ & $\begin{array}{r}<40 \\
160\end{array}$ & $\begin{array}{l}<40 \\
<40\end{array}$ & $\begin{array}{l}<40 \\
<40\end{array}$ \\
\hline $\begin{array}{l}\text { A/Sing. } \\
\text { (H1N1) }\end{array}$ & $\begin{array}{l}1 . \\
2 .\end{array}$ & $\begin{array}{l}<40 \\
<40\end{array}$ & $\begin{array}{l}<40 \\
<40\end{array}$ & $\begin{array}{l}<40 \\
\cdot 40\end{array}$ & $\begin{array}{r}<40 \\
640\end{array}$ & $\begin{array}{l}<40 \\
<40\end{array}$ & $\begin{array}{l}<40 \\
<40\end{array}$ \\
\hline B/USSR & $\begin{array}{l}1 . \\
2 .\end{array}$ & $\begin{array}{l}<40 \\
<40\end{array}$ & $\begin{array}{l}<40 \\
<40\end{array}$ & $\begin{array}{l}<40 \\
<40\end{array}$ & $\begin{array}{l}<40 \\
<40\end{array}$ & $\begin{array}{l}<40 \\
2560\end{array}$ & $\begin{array}{r}<40 \\
640\end{array}$ \\
\hline$B / A A$ & $\begin{array}{l}1 . \\
2 .\end{array}$ & $\begin{array}{l}<40 \\
<40\end{array}$ & $\begin{array}{l}<40 \\
<40\end{array}$ & $\begin{array}{l}<40 \\
<40\end{array}$ & $\begin{array}{l}<40 \\
<40\end{array}$ & $\begin{array}{r}<40 \\
640\end{array}$ & $\begin{array}{l}<40 \\
2560\end{array}$ \\
\hline
\end{tabular}

Tab. 2: Hämagglutinationshemmende Antikörper (Huhn) im Kreuz-Hämagglutinationshemmtest

1. = vor Immunisierung

2. = 45-53 Tage nach Immunisierungsbeginn

\begin{tabular}{|c|c|c|c|c|c|c|c|}
\hline \multirow{2}{*}{$\begin{array}{l}\text { Antiserum } \\
\text { (Huhn) }\end{array}$} & \multirow{2}{*}{$\begin{array}{l}\text { *Ab- } \\
\text { nahme }\end{array}$} & \multicolumn{3}{|c|}{ Referenzstämme } & \multicolumn{2}{|l|}{. } & \multirow[b]{2}{*}{ B/AA } \\
\hline & & $\begin{array}{l}\text { A/Miss. } \\
\text { (H3N2) }\end{array}$ & $\begin{array}{l}\text { A/Leningr. } \\
\text { (H3N2) }\end{array}$ & $\begin{array}{l}\text { A/Chile } \\
\text { (H1N1) }\end{array}$ & $\begin{array}{l}\text { A/Sing. } \\
\text { (H1N1) }\end{array}$ & B/USSR & \\
\hline $\begin{array}{l}\text { A/Miss. } \\
\text { (H3N2) }\end{array}$ & $\begin{array}{l}1 . \\
2 .\end{array}$ & $\begin{array}{r}<8 \\
1024\end{array}$ & $\begin{array}{l}<8 \\
256\end{array}$ & $\begin{array}{l}<8 \\
<8\end{array}$ & $\begin{array}{l}<8 \\
<8\end{array}$ & $\begin{array}{l}<8 \\
<8\end{array}$ & $\begin{array}{l}<8 \\
<8\end{array}$ \\
\hline $\begin{array}{l}\text { A/Leningr. } \\
\text { (H3N2) }\end{array}$ & $\begin{array}{l}1 . \\
2 .\end{array}$ & $\begin{array}{l}<8 \\
512\end{array}$ & $\begin{array}{r}16 \\
512\end{array}$ & $\begin{array}{l}<8 \\
<8\end{array}$ & $\begin{array}{l}<8 \\
<8\end{array}$ & $\begin{array}{l}<8 \\
<8\end{array}$ & $\begin{array}{l}<8 \\
<8\end{array}$ \\
\hline $\begin{array}{l}\text { A/Chile } \\
\text { (H1N1) }\end{array}$ & $\begin{array}{l}1 . \\
2 .\end{array}$ & $\begin{array}{l}<8 \\
<8\end{array}$ & $\begin{array}{l}16 \\
16\end{array}$ & $\begin{array}{l}<8 \\
128\end{array}$ & $\begin{array}{r}<8 \\
16\end{array}$ & $\begin{array}{l}<8 \\
<8\end{array}$ & $\begin{array}{l}<8 \\
<8\end{array}$ \\
\hline $\begin{array}{l}\text { A. Sing. } \\
\text { (H1N1) }\end{array}$ & $\begin{array}{l}1 . \\
2 .\end{array}$ & $\begin{array}{l}<8 \\
<8\end{array}$ & $\begin{array}{l}32 \\
32\end{array}$ & $\begin{array}{r}<8 \\
8\end{array}$ & $\begin{array}{l}<8 \\
128\end{array}$ & $\begin{array}{l}<8 \\
<8\end{array}$ & $\begin{array}{l}<8 \\
<8\end{array}$ \\
\hline B/USSR & $\begin{array}{l}1 . \\
2 .\end{array}$ & $\begin{array}{l}<8 \\
<8\end{array}$ & $\begin{array}{l}32 \\
32\end{array}$ & $\begin{array}{l}<8 \\
<8\end{array}$ & $\begin{array}{l}<8 \\
<8\end{array}$ & $\begin{array}{r}<8 \\
1024\end{array}$ & $\begin{array}{l}<8 \\
256\end{array}$ \\
\hline$B / A A$ & $\begin{array}{l}1 . \\
2 .\end{array}$ & $\begin{array}{l}<8 \\
<8\end{array}$ & $\begin{array}{r}<8 \\
8\end{array}$ & $\begin{array}{l}<8 \\
<8\end{array}$ & $\begin{array}{l}<8 \\
<8\end{array}$ & $\begin{array}{r}<8 \\
64\end{array}$ & $\begin{array}{r}<8 \\
1024\end{array}$ \\
\hline
\end{tabular}



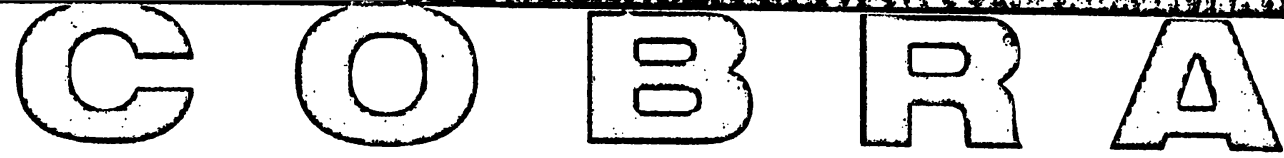

\section{der leistungsfähige Gammaprobenwechsler für RIA}

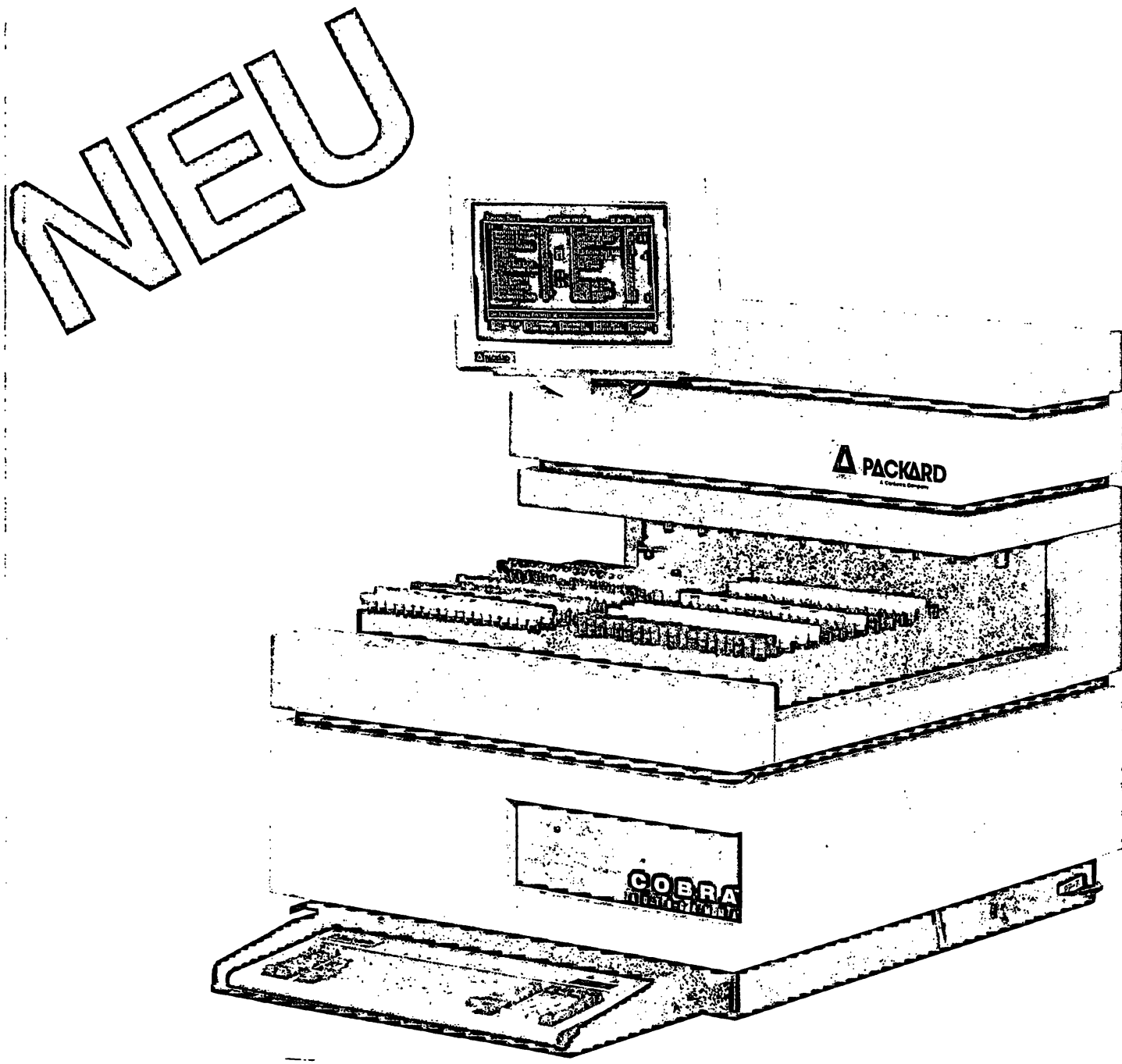

Sie suchen ein Gerät für einfache und schnelle RIA-Analysen.

Hier ist es... COBRA für höchsten Probendurchsatz und gröBte MeBgenauigkeit.

- Multidetektorgerät mit 5 oder $10 \mathrm{Nal}$ Detektoren

- Oder Eindetektorgerät mit 2" oder 3" Nal Detektor

- Probenwechsler für 1000 Proben

- 60 RIA/IRMA Protokolle

- Systemkontrolle durch PC

- Automatische Kalibrierung

- Einfachste Bedienung
- RIASmart Software für höchste Flexibilität bei der Auswertung

- Expert-QC Qualitätskontrolle für 12 Parameter und 180 Assayanalysen

- Umfassende Kurvenkalkulationssoftware

- Software für alle Assayanforderungen

- Spectraview für Spektrendarstellung

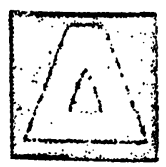

\section{CANIIERRA PACKARD}




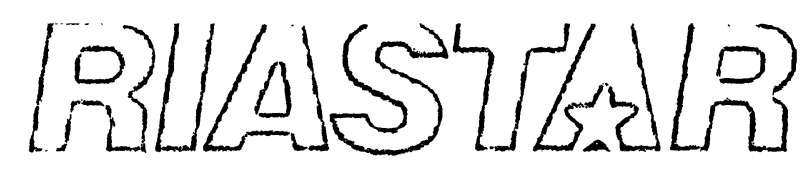

\section{der manuelle RIA-Meßplatz mit dem optimalen Preis-Leistungs-Verhältnis}

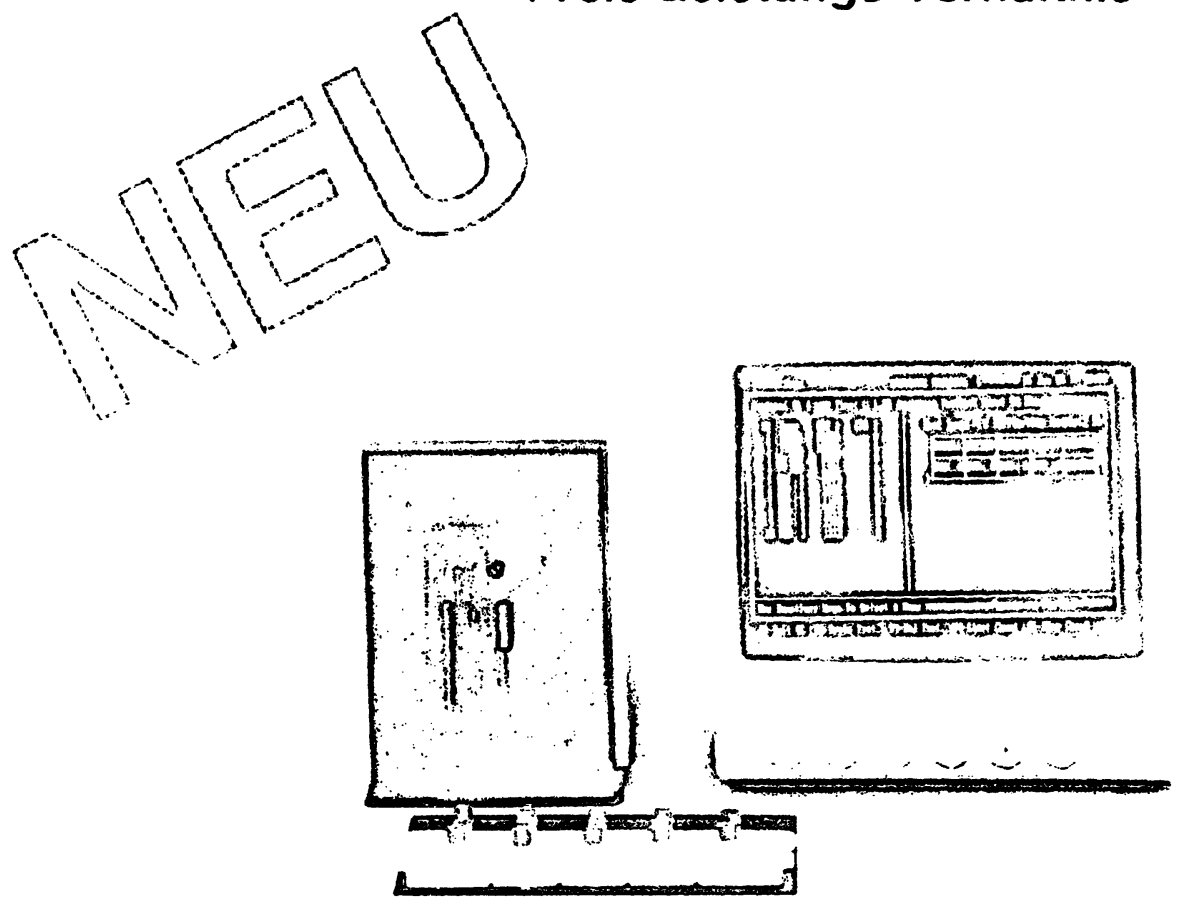

MUEDICA88 MESSE DÜSSELDORF

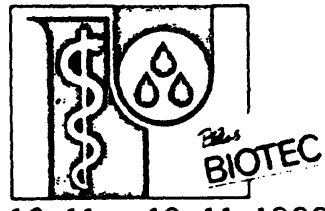

16. 11. - 19. 11. 1988

Halle $4 \cdot$ Stand $4 \mathrm{H} 47$

\section{RLASTAR}

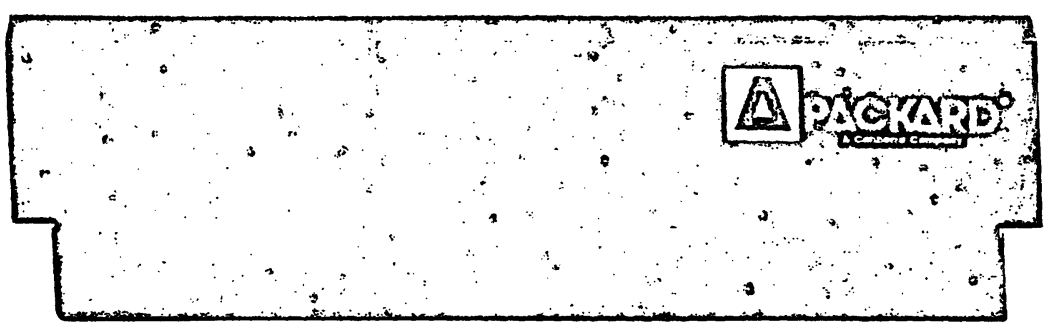

Sie suchen ein Gerät, das zuverlässig, schnell und preiswert ist.

Hier ist es . . der RIASTAR von Packard.

- wahlweise 5. 10 oder 20 Detektoren

- RIASmart Software für alle Assayanforderungen

- Expert QC Qualitätskontrolle für 12 Parameter und 180 Assayanalysen

- RIASTAR Express Protokollkassette für automatische Messungen und Auswertungen
- automatische Kalibrierung

- Systemkontrolle durch PC

- Auto-Start Sensor

- integrierter PC

- on-line Spektrendarstellung durch Vielkanalertechnologie 
wohl im IgG-IFTE als auch im $\mathrm{HAH}$ zu beobachten, jedoch nicht im IgA-IFTE. Das Serumpaar des Patienten 2 zeigte im IgG-IFTE mit beiden H1N1-Stämmen einen signifikanten Titeranstieg von $<320$ auf 1280 , auch im $\mathrm{HAH}$ zeigte das Zweitserum mit beiden Influenza A/ H1 N1-Stämmen einen Anstieg auf 32, so daß hier weder auf Grund des HAH noch des IFTE der infizierende Stamm vermutet werden kann, dasselbe gilt für die Seren von Patient 4 und Patient 10. Das Serumpaar von Patient 3 zeigt im IgG-IFTE einen signifikanten Titeranstieg sowohl mit A/Chile als auch mit A/Sing., wobei mit A/Sing. ein höherer Titer von 5120 erhalten wurde. Im Gegensatz dazu waren die $\mathrm{HAH}$-Titer im Zweitserum mit beiden Influenza A/H1N1 -Stämmen mit 64 gleich hoch. Die Seren der Patienten 5 und 7 zeigten im $\lg$ - und IgA-IFTE gleich hohe Titer, so daß keine Stammspezifität festgestellt werden konnte. Im Gegensatz hierzu wies im $\mathrm{HAH}$ das Serum von Patient 5 einen Titer von 256 mit A/Sing. auf, mit A/Chile hingegen nur von 64. Bei dem Serum von Patient 7 zeigte der $\mathrm{HAH}$ einen Titer > 1024 mit A/ Chile und mit A/Sing. einen geringeren Titer von 256. Bei Patient 8 hingegen sind die $\mathrm{HAH}$-Titer gleich hoch, im Gegensatz zu den IFTE-Titer. Von den 11 geprüften $\mathrm{Pa}$ tientenseren zeigten 2 nur mit dem $\mathrm{HAH}, 6$ mit $\mathrm{HAH}$ und IFTE und 3 nur mit dem IFTE stammspezifische Unterschiede.

Zur weiteren Überprüfung der Möglichkeit einer stammspezifischen Diagnostik mittels des IFTEs wurden $16 \mathrm{Se}$ rumpaare von Patienten mit einer durch Virusisolierung und/oder durch einen signifikanten Titeranstieg in der KBR bzw. im IFTE gesicherten Influenza A/H3N2-Infektion untersucht. Alle Patienten waren Anfang 1983 erkrankt gewesen. Zu dieser Zeit kursierte ein A/Bangkok/ 1/79-ähnlicher Influenzastamm. Die Ergebnisse sind auf Tab. 4 dargestellt. Die Untersuchung dieser Seren mit dem erst 1986 isolierten Stamm A/Leningr. ergab, daß 6 (Patienten 1, 2, 12, 14, 15, 16) der 16 Patienten einen signifikanten Tieranstieg im IgG - und 2 Patienten ( $\mathrm{Pa}$ tienten 2, 11) im IgA-Bereich zeigten. Im IgM-Bereich war keine Immunantwort mit $A /$ Leningr. nachweisbar (Patienten 13, 15, 16). Im Vergleich zu den Ergebnissen mit dem zum Erkrankungszeitraum aktuellen Stamm A/ Bangkok/1/79 reagierten alle Seren im IFT mit dem 7 Jahre später kursierenden Stamm A/Leningr. mit einem deutlich niedrigeren Titer. Im $\mathrm{HAH}$ waren diese Unterschiede weniger deutlich, da die Seren mit A/Leningr. nur um eine Titerstufe niedriger reagierten, außer bei Patienten 1, 3, 6 und 15, bei denen kein Unterschied in der Reaktivität mit A/Leningr. oder A/Bang. zu beobachten war. Die Seren des Patienten 9 zeigten im $\mathrm{HAH}$ einen 4 fachen Titerunterschied. Die Ergebnisse der 16 Patienten zeigten im IFTE deutliche stammspezifische Titerunterschiede, und zwar im IgG- wie im IgA-Bereich. Dagegen waren im $\mathrm{HAH}$ nur bei Seren von 10 Patienten Titerunterschiede, meist nur von einer Titerstufe, erkennbar. Die Spezifität beider Methoden ist somit vergleichbar.

\section{Diskussion}

Es ist davon auszugehen, daß HAH wie IFTE ausschließlich, bzw. weit überwiegend Antikörper gegen das Hämagglutinin des Influenzavirus messen und somit in erheblichem Grade stammspezifisch reagieren. Andererseits sind diese Methoden technisch so verschieden, daß man vermuten darf, daß sie zu unterschiedlichen Ergebnissen führen. Es sollte deshalb untersucht werden, inwieweit die Ergebnisse des IFTE hinsichtlich seiner Stammspezifität mit denen des $\mathrm{HAH}$ vergleichbar sind. Nach Abklärung der Reaktionen mit spezifischen Antiseren sollten die Teste anhand von Patientenseren, bei denen schwer übersichtliche anamnestische Reaktionen zu erwarten waren, verglichen werden.

Tab. 3: Prüfung der Immunantwort bei Seren von Patienten mit Influenza A/H1N1-Infektionen: Vergleich von KBR, IFTE und HAH

\begin{tabular}{|c|c|c|c|c|c|c|c|c|c|c|c|}
\hline \multirow{3}{*}{$\begin{array}{l}\text { Pat.- } \\
\text { Nr. }\end{array}$} & \multirow[t]{3}{*}{ Alter } & \multirow[t]{3}{*}{ Tag* } & \multirow{3}{*}{$\begin{array}{l}\text { KBR- } \\
\text { Titer }\end{array}$} & \multicolumn{6}{|c|}{ IFTE-Titer } & \multicolumn{2}{|l|}{ HAH-Titer } \\
\hline & & & & \multicolumn{3}{|c|}{ A/Chile/1/83 } & \multicolumn{3}{|c|}{ A/Sing./6/86 } & \multirow[t]{2}{*}{ A/Chile/1/83 } & \multirow[t]{2}{*}{ A/Sing. $/ 6 / 8$} \\
\hline & & & & $\lg \mathbf{G}$ & $\lg \mathbf{A}$ & $\lg M$ & $\lg G$ & $\lg A$ & $\lg M$ & & \\
\hline 1 & 86 & 20 & 640 & 5120 & $<40$ & $<40$ & 10240 & 1280 & $<40$ & 128 & $>1024$ \\
\hline $2^{\circ}$ & 36 & $\begin{array}{r}1 \\
13\end{array}$ & $\begin{array}{r}5 \\
40\end{array}$ & $\begin{array}{r}<320 \\
1280\end{array}$ & $\begin{array}{l}<40 \\
<40\end{array}$ & $\begin{array}{l}<40 \\
<40\end{array}$ & $\begin{array}{r}<320 \\
1280\end{array}$ & $\begin{array}{l}<40 \\
<40\end{array}$ & $\begin{array}{l}<40 \\
<40\end{array}$ & $\begin{array}{l}16 \\
32\end{array}$ & $\begin{array}{r}<4 \\
32\end{array}$ \\
\hline 3 & 28 & $\begin{array}{r}5 \\
14\end{array}$ & $\begin{array}{l}10 \\
20\end{array}$ & $\begin{array}{r}320 \\
1280\end{array}$ & $\begin{array}{l}<40 \\
<40\end{array}$ & $\begin{array}{l}<40 \\
<40\end{array}$ & $\begin{array}{r}640 \\
5120\end{array}$ & $\begin{array}{r}<40 \\
40\end{array}$ & $\begin{array}{l}<40 \\
<40\end{array}$ & $\begin{array}{r}64 \\
-\quad 64\end{array}$ & $\begin{array}{r}4 \\
64\end{array}$ \\
\hline 4 & 32 & 15 & 40 & 2560 & $<40$ & $<40$ & 1280 & $<40$ & $<40$ & 32 & 32 \\
\hline $5^{*}$ & 31 & 11 & 40 & 5120 & 40 & $<40$ & 5120 & 40 & $<40$ & 64 & 256 \\
\hline 6 & 36 & 7 & 10 & 320 & $<40$ & $<40$ & 1280 & $<40$ & $<40$ & 32 & 64 \\
\hline 7 & n.b. & n.b. & 80 & 20480 & 2560 & $<40$ & 20480 & 2560 & $<40$ & $>1024$ & 256 \\
\hline 8 & n.b. & n.b. & 80 & 10240 & 40 & $<40$ & 5120 & $<40$ & $<40$ & 128 & 128 \\
\hline 9 & n.b. & n.b. & 80 & 10240 & 160 & $<40$ & 5120 & 80 & $<40$ & 128 & 32 \\
\hline 10 & n.b. & n.b. & 20 & 1280 & $<40$ & $<40$ & 1280 & 40 & $<40$ & $<4$ & $<4$ \\
\hline 11 & n.b. & n.b. & 20 & 1280 & 40 & $<40$ & 640 & 40 & $<40$ & 8 & $<4$ \\
\hline
\end{tabular}

- Tag * Tag nach Auftreten der klinischen Symptome.

n.b. = nicht bekannt.

$\because$ - Bei diesen Patienten wurde Influenza A/H1N1 aus dem Nasen-Rachensekret isoliert. 
Die Reaktionen mit Influenza A/Chile (H1N1) und dem im August 1986 isolierten Influenza A/Sing. (H1N1) waren besonders interessant, da mit spezifischen Antiseren aus Frettchen im HAH keine (10) bzw. nur geringe (8) Kreuzreaktionen gefunden worden waren. Entsprechende Beobachtungen machten wir sowohl im $\mathrm{HAH}$ als auch im IFTE mit Seren immunisierter Hühner. Zwischen den beiden Influenza A/H1 N1 -Stämmen war die serologische Verwandtschaft gering. Auffallend war, daß das anti-A/ Chile-Serum (anti-H1) im IFTE auch mit A/Miss. (H3) einen Titer von 40 aufwies, ebenso zeigte das anti-A/ Miss.-Serum (anti-H3) mit A/Sing. (H1) einen Titer von 40. Hierfür ist folgende Erklärung möglich: Der IFTE zeigt - weil er empfindlicher ist als der $\mathrm{HAH}$ - bei niedrigen Serumverdünnungen auch eine typenspezifische Immunantwort an. Hierbei kann es sich um die Erkennung von konservierten Determinanten im Hämagglutinin, von gemeinsamen Antigendeterminanten an der Neuramini- dase, und sogar von Innenantigenen handeln. Ein Vergleich mit den Ergebnissen anderer Autoren ist uns nicht möglich, da uns keine neuere Literatur bekannt ist, in der Influenza A/H1N1 - und Influenza A/H3N2-Stämme untereinander verglichen wurden.

Bei der Untersuchung der anti-A/Leningr. (anti-H3)und anti-A/Miss. (anti-H3)-Hühnerseren entsprachen unsere Ergebnisse im $\mathrm{HAH}$ den Ergebnissen von dem Center for Disease Control der USA (9). Der IFTE differenzierte die Stämme ebenso gut. Der Verwandtschaftsgrad der beiden Influenzastämme war hoch. Darüber hinaus war aufgefallen, daß im HAH im Gegensatz zum IFTE unspezifische Reaktionen mit $A /$ Leningr. bereits mit dem Präserum zu beobachten waren. Diese Inhibitoren konnten durch zusätzliche Inaktivierung der Seren mit Neuraminidase von $\mathrm{V}$. cholerae neben der Behandlung mit $\mathrm{Na}$ Perjodat nicht eliminiert werden. Für die beiden Influen-

Tab. 4: Prüfung der Immunantwort bei Seren von Patienten mit einer gesicherten Influenza A/H3N2-Infektion: Vergleich von KBR, IFTE und $H A H$

\begin{tabular}{|c|c|c|c|c|c|c|c|c|c|c|c|}
\hline \multirow{3}{*}{$\begin{array}{l}\text { Pat.- } \\
\text { Nr. }\end{array}$} & \multirow[t]{3}{*}{ Alter } & \multirow[t]{3}{*}{ Tag* } & \multirow{3}{*}{$\begin{array}{l}\text { KBR- } \\
\text { Titer }\end{array}$} & \multicolumn{6}{|c|}{ IFTE-Titer } & \multicolumn{2}{|l|}{ HAH-Titer } \\
\hline & & & & \multicolumn{3}{|c|}{ A/Bangkok/1/79 } & \multicolumn{3}{|c|}{ A/Leningr./360/86 } & \multirow{2}{*}{$\begin{array}{l}\text { A/Bangkok/1/ } \\
79\end{array}$} & \multirow{2}{*}{$\begin{array}{l}\text { A/Leningr./ } \\
360 / 86\end{array}$} \\
\hline & & & & $\lg \mathbf{G}$ & $\lg A$ & $\lg M$ & $\lg \mathbf{G}$ & $\lg A$ & $\lg M$ & & \\
\hline $1^{\cdots}$ & 88 & $\begin{array}{r}0 \\
24\end{array}$ & $\begin{array}{r}5 \\
80\end{array}$ & $\begin{array}{r}<320 \\
2560\end{array}$ & $\begin{array}{r}<40 \\
320\end{array}$ & $\begin{array}{l}<40 \\
<40\end{array}$ & $\begin{array}{l}<320 \\
1280\end{array}$ & $\begin{array}{l}<40 \\
<40\end{array}$ & $\begin{array}{l}<40 \\
<40\end{array}$ & $\begin{array}{l}<8 \\
128\end{array}$ & $\begin{array}{l}<8 \\
128\end{array}$ \\
\hline $2^{\bullet}$ & 25 & $\begin{array}{r}0 \\
21\end{array}$ & $\begin{array}{r}20 \\
160\end{array}$ & $\begin{array}{r}320 \\
10240\end{array}$ & $\begin{array}{r}40 \\
1280\end{array}$ & $\begin{array}{l}<40 \\
<40\end{array}$ & $\begin{array}{r}320 \\
1280\end{array}$ & $\begin{array}{r}<40 \\
160\end{array}$ & $\begin{array}{l}<40 \\
<40\end{array}$ & $\begin{array}{r}<8 \\
1024\end{array}$ & $\begin{array}{l}<8 \\
512\end{array}$ \\
\hline $3 \cdots$ & 43 & $\begin{array}{r}0 \\
21\end{array}$ & $\begin{array}{l}20 \\
20\end{array}$ & $\begin{array}{r}320 \\
1280\end{array}$ & $\begin{array}{r}<40 \\
320\end{array}$ & $\begin{array}{l}<40 \\
<40\end{array}$ & $\begin{array}{r}<320 \\
320\end{array}$ & $\begin{array}{l}<40 \\
<40\end{array}$ & $\begin{array}{l}<40 \\
<40\end{array}$ & $\begin{array}{r}16 \\
128\end{array}$ & $\begin{array}{r}8 \\
128\end{array}$ \\
\hline 4 & 52 & $\begin{array}{r}1 \\
16\end{array}$ & $\begin{array}{r}<5 \\
20\end{array}$ & $\begin{array}{r}<320 \\
1280\end{array}$ & $\begin{array}{r}<40 \\
160\end{array}$ & $\begin{array}{l}<40 \\
<40\end{array}$ & $\begin{array}{l}<320 \\
<320\end{array}$ & $\begin{array}{l}<40 \\
<40\end{array}$ & $\begin{array}{l}<40 \\
<40\end{array}$ & $\begin{array}{l}16 \\
16\end{array}$ & $\begin{array}{l}8 \\
8\end{array}$ \\
\hline $5 *$ & 35 & $\begin{array}{r}0 \\
16\end{array}$ & $\begin{array}{r}5 \\
20\end{array}$ & $\begin{array}{r}320 \\
1280\end{array}$ & $\begin{array}{r}<40 \\
160\end{array}$ & $\begin{array}{l}<40 \\
<40\end{array}$ & $\begin{array}{l}<320 \\
<320\end{array}$ & $\begin{array}{r}<40 \\
40\end{array}$ & $\begin{array}{l}<40 \\
<40\end{array}$ & $\begin{array}{r}<8 \\
64\end{array}$ & $\begin{array}{r}<8 \\
32\end{array}$ \\
\hline 6 & 46 & $\begin{array}{r}1 \\
22\end{array}$ & $\begin{array}{l}40 \\
40\end{array}$ & $\begin{array}{l}1280 \\
2560\end{array}$ & $\begin{array}{l}80 \\
80\end{array}$ & $\begin{array}{l}<40 \\
<40\end{array}$ & $\begin{array}{r}<320 \\
640\end{array}$ & $\begin{array}{l}<40 \\
<40\end{array}$ & $\begin{array}{l}<40 \\
<40\end{array}$ & $\begin{array}{l}64 \\
64\end{array}$ & $\begin{array}{l}64 \\
64\end{array}$ \\
\hline $7 * *$ & 32 & $\begin{array}{l}1 \\
8\end{array}$ & $\begin{array}{r}5 \\
40\end{array}$ & $\begin{array}{r}<320 \\
1280\end{array}$ & $\begin{array}{r}<40 \\
80\end{array}$ & $\begin{array}{l}<40 \\
<40\end{array}$ & $\begin{array}{r}<320 \\
320\end{array}$ & $\begin{array}{l}<40 \\
<40\end{array}$ & $\begin{array}{l}<40 \\
<40\end{array}$ & $\begin{array}{r}<8 \\
64\end{array}$ & $\begin{array}{l}16 \\
32\end{array}$ \\
\hline $8 *$ & 46 & 12 & 20 & 5120 & 160 & $<40$ & 320 & $<40$ & $<40$ & 128 & 64 \\
\hline $9 \cdots$ & 33 & 17 & 20 & 640 & 40 & $<40$ & $<320$ & $<40$ & $<40$ & 32 & 8 \\
\hline $10^{* *}$ & 52 & $\begin{array}{l}1 \\
9\end{array}$ & $\begin{array}{r}5 \\
20\end{array}$ & $\begin{array}{r}<320 \\
2560\end{array}$ & $\begin{array}{r}<40 \\
160\end{array}$ & $\begin{array}{l}<40 \\
<40\end{array}$ & $\begin{array}{r}<320 \\
320\end{array}$ & $\begin{array}{l}<40 \\
<40\end{array}$ & $\begin{array}{l}<40 \\
<40\end{array}$ & $\begin{array}{l}\text { n.b. } \\
\text { n.b. }\end{array}$ & $\begin{array}{l}\text { n.b. } \\
\text { n.b. }\end{array}$ \\
\hline 11 & 38 & $\begin{array}{r}3 \\
14\end{array}$ & $\begin{array}{r}5 \\
320\end{array}$ & $\begin{array}{r}320 \\
5120\end{array}$ & $\begin{array}{l}<40 \\
1280\end{array}$ & $\begin{array}{l}<40 \\
<40\end{array}$ & $\begin{array}{r}<320 \\
640\end{array}$ & $\begin{array}{c}<40 \\
160\end{array}$ & $\begin{array}{l}<40 \\
<40\end{array}$ & $\begin{array}{r}32 \\
128\end{array}$ & $\begin{array}{l}32 \\
64\end{array}$ \\
\hline 12 & 68 & $\begin{array}{r}1 \\
16\end{array}$ & $\begin{array}{r}40 \\
160\end{array}$ & $\begin{array}{r}<320 \\
20480\end{array}$ & $\begin{array}{r}<40 \\
640\end{array}$ & $\begin{array}{r}<40 \\
40\end{array}$ & $\begin{array}{r}<320 \\
2560\end{array}$ & $\begin{array}{l}<40 \\
<40\end{array}$ & $\begin{array}{r}<40 \\
40\end{array}$ & $\begin{array}{r}64 \\
512\end{array}$ & $\begin{array}{r}64 \\
256\end{array}$ \\
\hline 13 & 33 & $\begin{array}{r}3 \\
14\end{array}$ & $\begin{array}{r}5 \\
40\end{array}$ & $\begin{array}{r}320 \\
2560\end{array}$ & $\begin{array}{r}<40 \\
160\end{array}$ & $\begin{array}{r}<40 \\
160\end{array}$ & $\begin{array}{r}<320 \\
640\end{array}$ & $\begin{array}{l}<40 \\
<40\end{array}$ & $\begin{array}{l}<40 \\
<40\end{array}$ & $\begin{array}{l}256 \\
256\end{array}$ & $\begin{array}{l}32 \\
64\end{array}$ \\
\hline 14 & 60 & $\begin{array}{r}1 \\
10\end{array}$ & $\begin{array}{r}<5 \\
80\end{array}$ & $\begin{array}{r}320 \\
10240\end{array}$ & $\begin{array}{r}<40 \\
640\end{array}$ & $\begin{array}{r}<40 \\
40\end{array}$ & $\begin{array}{r}<320 \\
1280\end{array}$ & $\begin{array}{l}<40 \\
<40\end{array}$ & $\begin{array}{l}<40 \\
<40\end{array}$ & $\begin{array}{r}8 \\
128\end{array}$ & $\begin{array}{r}8 \\
64\end{array}$ \\
\hline 15 & n.b. & $\begin{array}{l}\text { n.b. } \\
\text { n.b. }\end{array}$ & $\begin{array}{l}10 \\
40\end{array}$ & $\begin{array}{r}640 \\
10240\end{array}$ & $\begin{array}{r}<40 \\
160\end{array}$ & $\begin{array}{r}<40 \\
160\end{array}$ & $\begin{array}{r}<320 \\
1280\end{array}$ & $\begin{array}{l}<40 \\
<40\end{array}$ & $\begin{array}{l}<40 \\
<40\end{array}$ & $\begin{array}{l}<8 \\
256\end{array}$ & $\begin{array}{l}<8 \\
256\end{array}$ \\
\hline 16 & 79 & $\begin{array}{r}3 \\
14\end{array}$ & $\begin{array}{r}20 \\
320\end{array}$ & $\begin{array}{r}2560 \\
81920\end{array}$ & $\begin{array}{r}80 \\
1280\end{array}$ & $\begin{array}{r}<40 \\
160\end{array}$ & $\begin{array}{r}<320 \\
20480\end{array}$ & $\begin{array}{r}<40 \\
80\end{array}$ & $\begin{array}{l}<40 \\
<40\end{array}$ & $\begin{array}{r}64 \\
256\end{array}$ & $\begin{array}{r}32 \\
128\end{array}$ \\
\hline
\end{tabular}

- Tag = Tag nach Auftreten der klinischen Symptome.

n.b. = nicht bekannt.

$\because=$ Bei diesen Patienten wurde Influenza A/H3N2 aus dem Nasen-Rachensekret isoliert. 
za B-Stämme ergab sich im HAH und IFTE ein ähnlicher Verwandtschaftsgrad, der wiederum dem aus der Literatur bekannten entsprach (5).

Die Influenza-Aktivität war in unserem Einzugsgebiet Anfang 1987 so gering, daß wir nur 13 Seren von 11 Patienten mit frischen Infektionen untersuchen konnten. Doch war an dieser geringen Probandenzahl schon ersichtlich, daß eine stammspezifische Influenza-Diagnostik, selbst wenn so große antigene Unterschiede zwischen den Stämmen zu erwarten waren wie in diesem Jahr, nur begrenzt möglich ist. Bei 6 Patienten mit Influenza $A / H 1 N 1-I n f e k t i o n e n$ bestand Übereinstimmung zwischen IFTE und $\mathrm{HAH}$, wohingegen wir mit beiden Testsystemen bei einem Patienten keinen Hinweis auf den infizierenden Influenzastamm erhielten. Bei den Seren von zwei anderen Patienten differenzierte der $\mathrm{HAH}$ besser als der IFTE. Der IFTE zeigte im Gegensatz zum HAH mit beiden Influenzastämmen gleich hohe Titer. Die Beurteilung der Ergebnisse ist bei diesen Patienten besonders schwierig oder gar unmöglich, da wir aus unserem Einzugsbereich kein Virus isoliert haben. Es wurde jedoch berichtet (6), daß die meisten Influenzafälle in diesem Zeitraum durch Influenza A/H1 N1 -Viren verursacht waren, die der neuen Variante $A /$ Singapore/6/86 nahestanden.

Wurden die H1N1-Untersuchungen mit zwei zeitlich dicht aufeinanderfolgenden Stämmen mit Patientenseren aus der Zeit, in der diese Stämme kursierten, so daß die Möglichkeit der Beeinflussung der Ergebnisse durch anamnestische Reaktionen und das Kursieren von Übergangsstämmen kompliziert sein konnte, durchgeführt, so wurden in der zweiten Versuchsreihe Patientenseren aus einer Epidemie, die sieben Jahre vor der Isolierung des heterologen Stammes lag, verwendet. Hier sollten also Unterschiede zwischen den Methoden, die durch Kreuzreaktionen bedingt sind, noch besser ersichtlich werden. In der Literatur wurden folgende Verwandtschaftsgrade zwischen den hier verwendeten Stämmen im HAH beschrieben (9): anti-A/Bang. (aus Frettchen) mit dem homologen Stamm 1:1280 und mit $A /$ Leningr. 1:80. Tab. 4 zeigt jedoch, daß in unseren Patientenseren mit dem $\mathrm{HAH}$ - wenn überhaupt - nur eine Differenz von 1 Titerstufe (Ausnahme Patient 9) bei Verwendung dieser zwei Stämme nachzuweisen war. Bei 4 von 16 Patientenserumpaaren konnten im HAH überhaupt keine Stammesunterschiede festgestellt werden. Mit dem IFTE wurden bei allen Patientenseren eine bevorzugte Antikörperantwort für den damals kursierenden Stamm gefunden. Wären diese Patientenseren aus dem Jahre 1983 nur mit A/Leningr., der damals noch nicht kursierte, untersucht worden, so wären im IFTE bei 4 von 16 und im HAH bei 8 von 16 Patientenpaaren eine falsche stammspezifische
Infektion angenommen worden. Bei dieser Untersuchung reagiert somit der IFTE deutlich stärker stammspezifisch. Die Ergebnisse legen den Schluß nahe, daß bei der Stamm-differenzierenden Influenza-Diagnostik der IFTE dem $\mathrm{HAH}$ überlegen ist. Bei der Untersuchung der $\mathrm{Pa}$ tientenseren wird deutlich, daß mit dem IFTE und dem $\mathrm{HAH}$ ein unterschiedliches Antikörperspektrum gemessen wird. Selbst wenn hier die Neuraminidase, die nur im IFTE eine Rolle spielen kann, aus quantitativen Gründen vermutlich nur eine untergeordnete Rolle spielt, so mißt doch der IFTE ausschließlich IgG (oder IgA oder IgM), während im HAH IgG und IgM und vielleicht konkurrierend auch IgA eine Rolle spielen könnten. Ein großer Vorteil des IFTE bleibt, daß unspezifische Seruminhibitoren keinen Einfluß auf den Test haben.

\section{Schrifttum:}

1. DOLLER, G., DOLLER, P. C., GERTH, H.-J.: Diagnostic significance of influenca subtype-specific $\operatorname{lgG}$, IgA, and $\lg M$ antibodies. J. Biol. Stand. 14, 163-175 (1986), 2. DÖLLER, G., DÓLLER, P. C., GERTH, H.-J.: Serologische Diagnostik von Influenza A- und B-Infektionen mit Hilfe der Komplementbindungsreaktion und der indirekten Immunfluoreszenztechnik. Lab.med. 10, 291 -296 (1986).

3. DOLLER, P. C., DOLLER, G., GERTH, H.-J.: Immunofluorescence test with antigen-loaded erythrocytes: detection of influenza virus specific $\lg G$, IgA and $\lg M$ antibodies. Med. Microbiol. Immunol. 173, 291 - 302 (1985).

4. DOWDLE, W. A., KENDAL, A. P., NOBEL, G. R.: Influenza viruses. In: LENNETTE, E. H., SCHMIDT, N. J. (eds.): Diagnostic Procedures for Viral, Rickettsial and Chlamydial Infections. 5th Edition. Washington, D.C., American Public Health Association: 585-609 (1979).

5. LANGE, W.: Influenza 1985/86. Bundesgesundhbl. 29, 332-334 (1986).

6. LANGE, W.: Influenza 1986/87. Bundesgesundhbl. 30, $321-322$ (1987).

7. LENNETTE, E. H., SCHMIDT, N. J.: Complement-fixation (CF) test. In: LENNETTE, E. H., SCHMIDT, N. J. (eds): Diagnostic Procedures for Viral, Rickettsial and Chlamydial Infections. 5th Edition, Washington, D.C., American Public Health Association: 35- 42 (1979).

8. MMWR: Antigenic variation of recent influenza $A(H 1 N 1)$ viruses. $35,510-512$ (1986).

9. MMWR: Antigenic variation of recent influenza $A(H 3 N 2)$ viruses. $37,38-39$ (1988).

\section{Danksagung}

Unser besonderer Dank gilt Herrn Dr. Bernd Stekeler für die Bereitstellung von Seren.

Anschriften der Verfasser:

Dr. G. Döller

Dr. H.-J. Gerth

Hygiene-Institut

Abteilung für Medizinische Virologie und Epidemiologie der

Viruskrankheiten

Silcherstraße 7

7400 Tübingen

Dr. C. Merk

Labor Dr. Koch - Dr. Merk

Schloßstraße 9

7955 Ochsenhausen 